\title{
STUDY OF LATE PHASE FLUORESCENCE IN THE RABBIT FUNDUS* $\dagger$
}

\author{
BY \\ A. W. SOLLOM AND I. A. R. BROWN \\ Birmingham and Midland Eye Hospital
}

NovotNY and Alvis (1961) were the first to photograph fluorescence in the ocular fundus after intravenous injection of fluorescein. They studied the characteristics of the passage of fluorescence through the fundus of the eye. These are now well established by serial photographs and cinematography; arterial, venous, and late phases being recognized. A number of ocular conditions have been studied clinically in which staining has persisted long after blood fluorescein levels have fallen below the visible threshold. Fluorescein has a small, water-soluble molecule which diffuses readily. It becomes bound to protein slowly and in insignificant amounts when injected intravenously. There is a rapid fall in the plasma concentration of fluorescein and within 10 minutes it has diffused into a volume four times that of the plasma (Dollery, Hodge, and Engel, 1962).

Current hypotheses of the phenomenon of late-phase staining are numerous. In their original paper, Novotny and Alvis (1961) suggested that the persistent mottled fluorescence, which they observed, is caused by a slow rate of fluorescein turnover in the choroid. It has also been postulated that the staining is attributable to extravasation of the water-soluble molecules in an area of tissue damage (Weidenthal and Plotkin, 1966). Retention of fluorescein fluorescence has been seen in patches of healed choroiditis and in areas of myopic chorio-retinal degeneration; Rubinstein and Paton (1966) considered that in these situations the visible fluorescein may be located in the sclera. Angioid streaks stain with fluorescein for some time after the retinal vessels become clear of the dye. Smith, Gass, and Justice (1964) assumed that this is due either to baring of the chorio-capillaris at the site of breaks in Bruch's membrane or to altered tissue permeability allowing extra-vascular retention of fluorescein for a longer period than elsewhere in the retinal tissues. In a study of diabetic retinopathy, Linhart, McIntosh, Heyman, and Hart (1964) thought that prolonged fluorescence, in this instance within vessels, might be due to staining of the walls of microaneurysms or possibly to a sluggish circulation in them. Ashton (1959) has pointed out that many diabetic exudates have an avascular centre, and fluorescein photographs show that many exudates do not fluoresce in the centre but only at the edges. It appears that increased diffusion of dye from damaged vessels is a characteristic feature of the vascular pathology associated with these lesions (Hodge and Dollery, 1964). Late fluorescence has also been attributed to increased visibility of choroidal

* Received for publication March 7, 1967.

† Address for reprints: Birmingham and Midland Eye Hospital, Church Street, Birmingham, 3.

$61 \mathrm{a}$

815 
fluorescence in such conditions as chloroquine retinopathy (Kearns and Hollenhorst, 1966) and drusen (Norton, Gass, Smith, Curtin, David, and Justice, 1965; Ernest and Krill, 1966), where there is a disturbance of the overlying pigment epithelium.

The present study is therefore directed at analysis of the late-staining phenomenon as seen in the fundi of rabbits damaged by trans-scleral diathermy. Staining characteristics of these lesions closely parallel the staining patterns of comparable lesions in the human fundus. It is, of course, appreciated that these findings may not explain all the types of late-phase fluorescence described above. The anatomy of the rabbit eye follows the normal human pattern except that in the retina the vessels pass laterally from the disc before dividing. There is a horizontal band of opaque nerve fibres on each side of the optic disc. Rods predominate in the retina, which has the normal layers but a scarcity of ganglion cells. The choroid is of typical mammalian type, similar to, but not as thick as, the choroid in humans (Prince, 1956).

\section{Method}

For this study rabbits weighing 2 to $3 \mathrm{~kg}$. are used. Albinos are excluded. In preparation for the diathermy procedure the pupils are fully dilated with cyclopentolate $0.5 \mathrm{per}$ cent. and atropine 1 per cent. drops. Uncomplicated general anaesthesia is regularly obtained by intravenous injection of $45 \mathrm{mg}$. (approx.) pentobarbitone sodium (Nembutal) supplemented by topical drops of amethocaine 1 per cent., the operating time being in the region of 10 minutes. Sclera is exposed through a short incision, dividing conjunctiva and Tenon's capsule, between the inferior and lateral rectus muscles. A single lesion is produced by surface diathermy beneath the inferior rectus and near the posterior pole. This is a good position for fundus photography and is free from both retinal vessels and medullated nerve fibres. Satisfactory diathermy lesions can be produced using a flat surface applicator with a current of 50-60 mA for 6 seconds. A reaction which is ideal for fundus photography is a clearly-defined round grey area about $2 \mathrm{~mm}$. in diameter. At the end of the operation drops of neomycin 0.5 per cent. are instilled, together with atropine 1 per cent. to facilitate re-examination. No sutures are required and there has been no post-operative infection.

Serial photography is programmed for the following 5 weeks. During this period colour photographs of the lesions (taken with a hand-held Kowa fundus camera with Kodachrome II film, ASA rating 25) are compared with fluorescence studies using a Zeiss fundus camera. We find that the best results for the latter are obtained from Kodak Tri-X film ASA rating 400, with a combination of Wratten Gelatine filters, No. 47 in the No. 7 aperture for excitation of fluorescence, and No. 58 before the camera. For fluorescein photography general anaesthesia is used. After induction with intravenous Nembutal $30 \mathrm{mg}$., anaesthesia is continued with 2 per cent. fluothane in air, by means of a Fluotec unit. This choice avoids the troublesome miosis which occurs when fluothane is used alone, despite previous instillation of atropine. When the depth of anaesthesia is inadequate, photography is difficult because of nystagmus and random drifting movements of the eyes. Good fluorescence is achieved by intravenous injection of $0.3 \mathrm{ml} .10$ per cent. aqueous fluorescein. Serial photographs are taken at 1 minute intervals from 5 to 10 minutes later. No exposures are made before a time lapse of 5 minutes because only late-phase fluorescence is being studied, the arterial and venous phases being ignored.

In this investigation a total of 21 fluorescein studies were made using twelve rabbits. The animals were not anaesthetized at intervals of less than 5 days. Some rabbits were reserved for study during the later weeks because of fear that mortality under anaesthesia 
would be high. Seven successful fluorescein studies were made during the first postoperative week, three in the second, four in the third, and four between the third and fifth weeks.

Examination of numerous colour photographs during this time showed that there was no significant variation in the healing processes and so fluorescein studies were limited to the above. Colour photographs of all animals confirmed the ophthalmoscopic impression that all lesions at each phase were virtually the same. Fluorescein studies were therefore limited to a few representative animals.

\section{Results}

The immediate diathermy reaction in the fundus is a uniform grey-white circular area of coagulation with a well-defined edge; 3 days later clumps of pigment form at the periphery, giving a mottled boundary to a grey central patch, and during the next 2 weeks pigment stippling spreads towards the centre, by which time less than a third of the remaining central area appears coagulated. Among the clumps of pigment some choroidal vessels are occasionally seen. After 4 weeks the central core of the lesion disappears and the whole area is uniformly mottled with pigment clumps over a depigmented background.

At intervals in the healing process, photographs of the lesion taken with a Kowa camera are compared with fluorescein studies made at the same stage.

During the first week the dense grey centre in the fundus photograph corresponds to an area which does not fluoresce. Outside this area the depigmented ring is found to fluoresce brightly, while the outer ring of pigment remains visible as a dark line (Fig. 1).

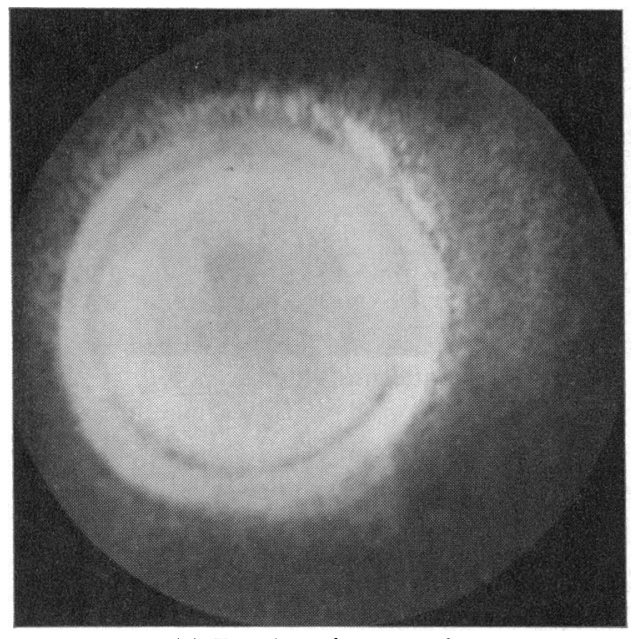

(a) Fundus photograph.

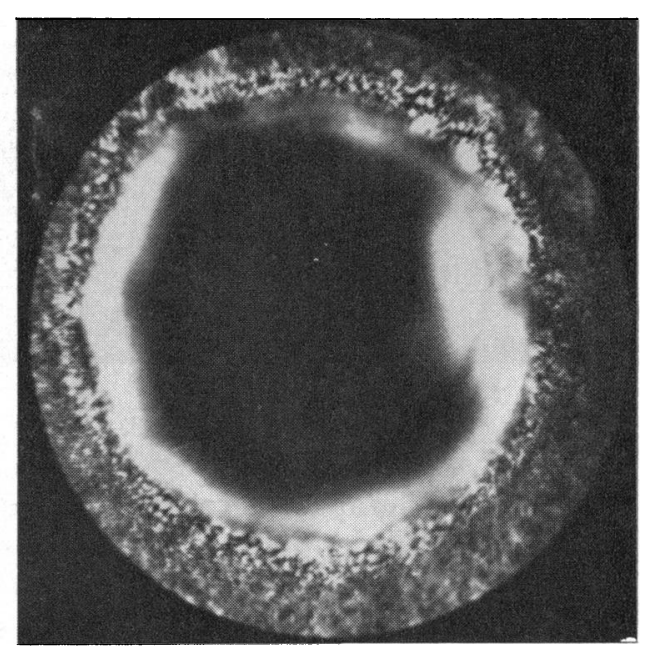

(b) Pattern of fluorescence.

Fig. 1.-Lesion during first week.

During the second and third weeks the spreading area of pigment stippling and depigmentation demonstrates bright late-phase fluorescence-the clumps of pigment forming black islands in the white fluorescent patch (Figs 2 and 3, overleaf). The central coagulated area remains dark but gradually decreases in size throughout the healing process (Fig. 4, overleaf). 




(a) Fundus photograph.

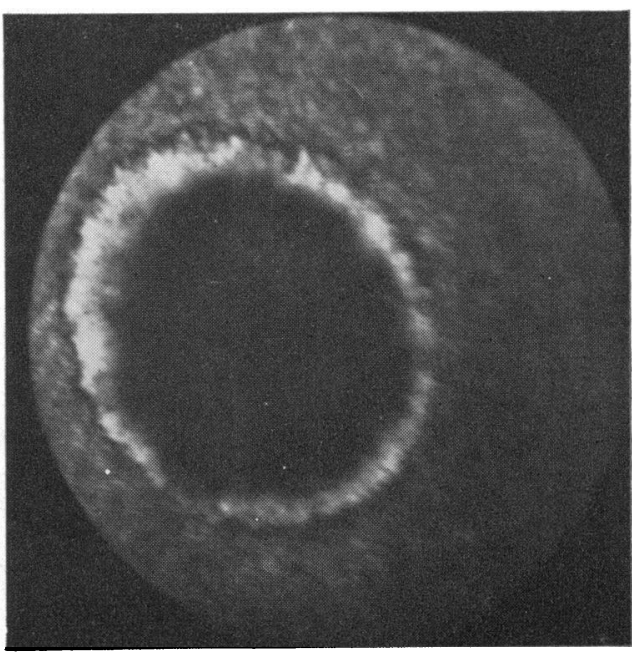

(b) Pattern of fluorescence.

Fig. 2.-Lesion during second week.



(a) Fundus photograph.

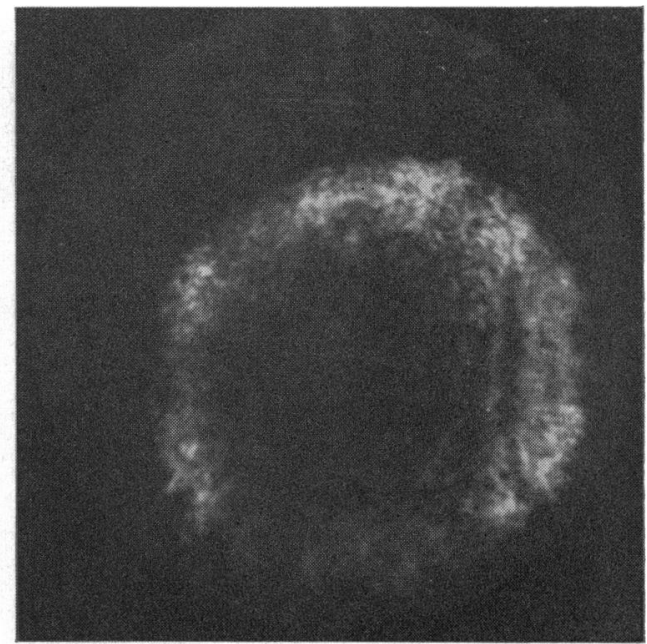

(b) Pattern of fluorescence.

Fig. 3.-Lesion during third week.

Serial photographs were taken in selected rabbits after intravenous fluorescein injection. The late-phase staining did not alter at all in the time between 5 and 15 minutes after injection, the photographs being identical. At all stages of healing the pattern of fluorescence corresponded with the depigmented area as seen by ophthalmoscopic examination and confirmed by colour photographs.

\section{Comment}

This series of experiments shows that it is possible to produce a lesion in the rabbit which retains fluorescein after intravenous injection. The method appears completely reliable and predictable; it is safe and easily performed. Late fluorescence as seen in these lesions does not appear to be associated with leakage from damaged 


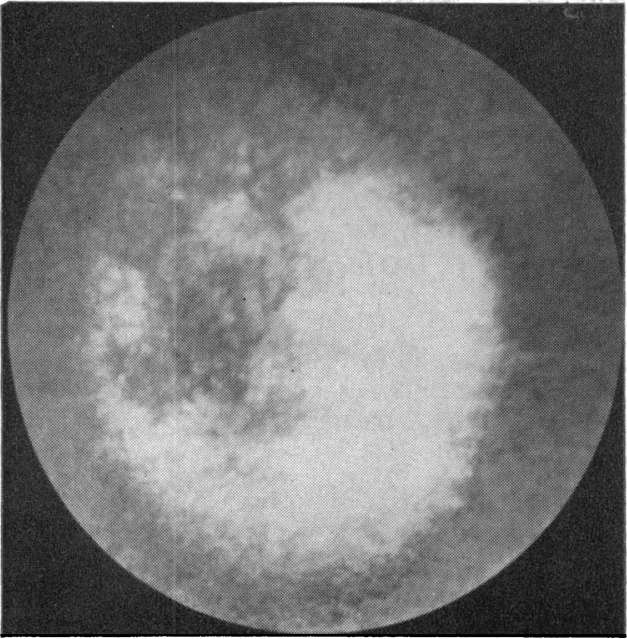

(a) Fundus photograph.

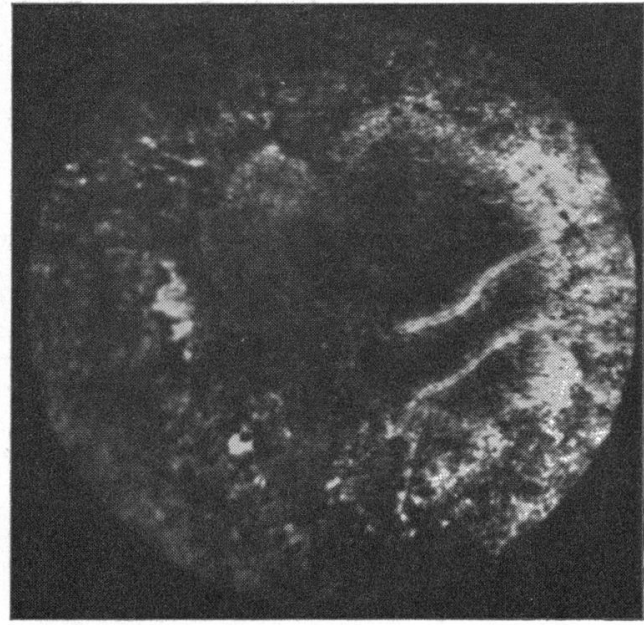

(b) Pattern of fluorescence.

FIG. 4.--Lesion during fifth week.

capillaries because this phenomenon would allow fluorescein to diffuse outwards as well as towards the centre of the early lesions. Photographs show that this is not the case. It seems unlikely that the fluorescence originates in the choroidal circulation, because it occurs in the white areas of choroidal destruction. It is our impression from the present study that fluorescein, diffusing slowly through the dense fibrous tissue of the sclera, may become visible when the normal pigment pattern is disturbed and the filtering effect of circulating haemoglobin in the chorio-capillaris is removed. The absence of fluorescence in the central area of these lesions may be explained by the screening effect of a haemorrhagic exudate in the choroid. It is, therefore, planned to extend the investigation and a further study has been designed to locate the exact anatomical site of the late-phase fluorescence in these diathermy lesions. Five minutes after fluorescein injection the stable state of late fluorescence has been reached-as has been amply shown in the present work. In the extension of this work the eye will be enucleated exactly 5 minutes after the same intravenous dose of fluorescein for microscopical study.

\section{Summary}

Fundus photography shows that fluorescein is retained in lesions produced by trans-scleral diathermy in the eyes of rabbits. Ophthalmoscopy showed that the healing process at the various stages was strictly comparable in all animals. The best placed lesions were selected for photography at regular-approximately weeklyintervals for 5 weeks. Late-phase fluorescence was consistently localized to the areas showing pigment disturbance. The findings are discussed and further work is outlined.

We are most grateful for the advice and encouragement given by Mr. P. Jameson Evans and Mr. K. Rubinstein of the Birmingham and Midland Eye Hospital; also to Prof. G. Slaney, Department of Surgery, Birmingham University. We should also like to thank Mr. T. F. Dee of the Photographic Department, Queen Elizabeth Hospital, Birmingham. 
Ashton, N. (1959). Lancet, 2, 625.

\section{REFERENCES}

DOLlery, C. T. (1964). Quart. J. Med., 33, 117.

, Hodge, J. V., and Engel, M. (1962). Brit. med. J., 2, 1210.

ERnest, J. T., and Krill, A. E. (1966). Amer. J. Ophthal., 62, 1.

HodGe, J. V., and Dollery, C. T. (1964). Quart. J. Med., 33, 117.

Kearns, T. P., and Hollenhorst, R. W. (1966). Arch. Ophthal. (Chicago), 76, 378.

Linhart, J. W., McIntosh, H. D., Heyman, A., and Hart, L. M. (1964). Circulation, $29,577$.

Norton, E. W. D., Gass, J. D. M., Smith, J. Lawton, Curtin, V. T., David, N., and Justice. J. (1965). Trans. Amer. Acad. Ophthal. Otolaryng., 69, 631.

Novotny, H. R., and Alvis, D. L. (1961). Circulation 24, 82.

Prince, J. H. (1956). "Comparative Anatomy of the Eye". Thomas, Springfield, Ill.

Rubenstein, K., and Paton, A. (1966). Trans. ophthal. Soc. U.K., 86, 139.

Smith, J. Lawton, Gass, J. D. M., and Justice, J., JR. (1964). Brit. J. Ophthal., 48, 517.

Weidenthal, D., and Plotkin, J. (1966). Arch. Ophthal. (Chigaco), 76, 716. 